\title{
WAVE HEATING IN MAGNETIC FLUX TUBES
}

\author{
Wolfgang Kalkofen \\ Harvard-Smithsonian Center for Astrophysics \\ 60 Garden Street, Cambridge, Massachussetts 02138
}

\begin{abstract}
The solar chromosphere is identified with the atmosphere inside magnetic flux tubes. In the quiet sun, the layers of the low and middle chromosphere are heated by compressive waves with periods mainly between $2 \mathrm{~min}$ and $4 \mathrm{~min}$. These long-period waves probably supply all the energy required for the heating of the quiet solar chromosphere.
\end{abstract}

\section{INTRODUCTION}

It is commonly assumed that if the chromosphere is heated by acoustic waves, as suggested by Biermann (1946) and Schwarzschild (1948), these waves must have periods shorter than the acoustic cutoff period. Consequently, practically all the work on chromospheric heating has been concerned with the so-called short-period waves (for a recent review, $c f$. Narain \& Ulmschneider 1989). However, there is no observational evidence showing shock heating by such waves. This led Cram (1987) to claim that there is no evidence from ground-based or space-based facilities for any shock heating in the solar atmosphere. In contrast to this pessimistic view of acoustic heating of the solar chromosphere this paper asserts that the solar chromosphere below the $7000^{\circ} \mathrm{K}$ level is heated by compressive waves forming shocks and that their periods are near the acoustic cutoff period.

\section{CHROMOSPHERIC MODEL AND OBSERVATIONS}

There is a close correspondence between magnetic filaments and regions that are bright in the resonance lines of $\mathrm{Ca}^{+}$and the core of $\mathrm{H} \alpha$ (Athay 1976; $c f$. also Foing \& Bonnet 1984), implying dissipation of mechanical energy in regions of strong vertical magnetic field. This correlation between magnetic field and excess emission extends throughout the chromosphere and into the transition region between chromosphere and corona (Athay). And it is twofold: All regions of increased vertical magnetic field are bright in the cores of $\mathrm{H} \alpha$ and the $\mathrm{K}$ line, and all regions that are bright correspond to peaks of vertical magnetic field strength (Zirin 1988). This one-to-one correspondence suggests that the chromosphere exists only in association with the magnetic field.

The small size of bright cell points (also called grains) and the pronounced tendency of brightenings to recur in the same location requires an organization of the waves that heat the medium by an underlying structure, most likely the small-scale, intense magnetic field.

The observations of chromospheric heating thus suggest a two-phase model of the outer solar atmosphere, consisting of gas inside magnetic flux tubes that is heated mechanically and of gas in the intertube medium that may receive little mechanical energy 
(Ayres 1981, Ayres \& Testerman 1981, Ayres et al. 1986, Kalkofen 1989).

Observations of chromospheric oscillations show that waves with typically $3 \mathrm{~min}$ period dissipate energy in the chromosphere. The wave periods seen in the $\mathrm{K}$ line by Liu (1974) show periods mainly between $2 \mathrm{~min}$ and $4 \mathrm{~min}$, with most periods longward of the acoustic cutoff period of about $3 \mathrm{~min}$. These waves have been observed: (1) in $\mathrm{H} \alpha$ by Orrall (1966) and Bhatnagar \& Tanaka (1972); (2) in the K line by Liu (1973, 1974), Cram (1974), and Damé (1984); (3) in the H line by Cram \& Damé (1983) and Damé (1984); and (4) in the continuum by Yudin (1968) at $3.3 \mathrm{~cm}$, by Simon \& Shimabukuro (1971) at $3.3 \mathrm{~mm}$ and $3.5 \mathrm{~mm}$, and by Lindsey \& Roellig (1987) at $350 \mu \mathrm{m}$ and $800 \mu \mathrm{m}$.

In the $\mathrm{K}$ line (Liu 1974) and in the $\mathrm{H}$ line (Cram \& Damé 1983) the waves produce initially a symmetrical intensity enhancement in the far line wings, which arise deep in the photosphere. This feature propagates towards the line center, i.e., outward in the atmosphere. When it reaches the line core, the profile becomes highly asymmetric: at the frequencies of the emission peaks $\mathrm{K}_{2}$ and $\mathrm{H}_{2}$, which are formed in the low chromosphere (cf. Fig. 1 of Vernazza et al. 1981, henceforth VAL81), the waves produce enhancements of only the blue peaks, $\mathrm{K}_{2 v}$ and $\mathrm{H}_{2 v}$, typically by a factor of 3 at $\mathrm{K}_{2 v}$.

The size of the area that oscillates is larger than the size of the heated region. At the height of formation of the $\mathrm{K}_{2 v}$ peak, the oscillations due to a wave extend over an area of the order of $2000 \mathrm{~km}$ to $4000 \mathrm{~km}$, whereas the heated area is only $1500 \mathrm{~km}$ or less in diameter. This may imply that a typical flux tube diameter at the height of formation of the $\mathrm{K}_{2}$ feature is of the order of $1500 \mathrm{~km}$ and that the atmosphere outside a tube oscillates in phase with the inside gas ( $c f$. Defouw 1976).

\section{WAVE ENERGY}

Liu (1974) estimated the wave energy flux from the rms velocity amplitude and the phase speed of the waves, i.e., the speed with which intensity enhancements in the K line propagate through the atmosphere, using an empirical model in order to relate the position of an intensity increase in the line profile to the position of the wave front in the atmosphere. Then, assuming that the waves were acoustic he calculated the group velocity and thus the energy flux. This flux, which may be uncertain by a large margin, falls short of the flux estimated by VAL81 (cf. their Table 29) by only a factor of about 2 . Therefore, the estimate of the energy flux allows the conclusion that the waves observed in the $\mathrm{K}$ line may carry all the energy needed for the heating of the low and middle chromosphere.

In order to judge whether the waves observed in the $\mathrm{H}$ line carry enough energy to meet the needs of the chromosphere, Cram \& Damé (1983) compared the average $\mathrm{H}$ line emission in their observations with the $\mathrm{H}$ line emission calculated from the empirical model of the average chromosphere (model C of Vernazza et al. 1981, henceforth VAL-C). They determined the observed emitted energy from the difference between the average profile of all their observations and the average profile of the lowest decile. Since the latter shows some evidence of heating, their estimate is less than the total dissipated mechanical energy. Their estimate gave $80 \%$ of the emission of the empirical model. Thus, one may conclude again that the heating of the layers in which the $\mathrm{H}$ line arises is consistent with heating by only these long-period waves.

Anderson \& Athay (1989) determined the dissipation rate of the chromospheric heating mechanism from the empirical temperature structure of the model VAL-C using a model atmosphere code. In addition to the familiar radiation terms present in radiative equilibrium the energy equation included a source term representing mechanical energy input. They matched the temperature curve of the empirical model in the layers of the temperature plateau below about $7000^{\circ} \mathrm{K}$ with a constant flux divergence per unit mass of $d F / \rho d x=4.5 \times 10^{9} \mathrm{erg}^{-1} \mathrm{~s}^{-1}$. In order to deduce the nature of the heating mechanism 
they assumed dissipation by acoustic waves, which implied a velocity amplitude of about half the sound speed, a result that is consistent with heating by shock waves. As further support for their hypothesis of acoustic wave heating they could point to the value of the microturbulent velocity in the same layers, which is approximately equal to the sound speed in these layers. Thus, the energy input into the chromosphere in the region of the temperature plateau below about $7000^{\circ} \mathrm{K}$ is consistent with the assumption of heating by compressional waves alone.

Weak-shock theory (cf. Ulmschneider 1970, Bray \& Loughhead 1974) permits an estimate of the wave period and the Mach number of the waves for shocks that have reached their limiting strength, where the growth of the wave amplitude due to the outward propagation in a gravitationally stratified gas is balanced by the decay of the amplitude due to dissipation. The theory is not directly applicable to the tube waves that heat the chromosphere since they are not plane and the shocks are not weak. But using the theory for a rough estimate of wave properties, the dissipation rate found by Anderson \& Athay implies that the shocks are fairly strong, with a Mach number of nearly 2 , and that the wave period is about $1 \mathrm{~min}$, not much different from the observed periods. These results thus are broadly consistent with heating of the chromosphere in the layers of the temperature plateau by compressional waves with periods near the acoustic cutoff period.

\section{WAVE PERIODS}

Acoustic waves in a gravitationally stratified medium propagate in the vertical direction only if their period is shorter than the acoustic cutoff period ( $c f$. Schatzman \& Souffrin 1967), approximately $3 \mathrm{~min}$ in the upper solar atmosphere. In deriving this result from the linearized equations of hydrodynamics in a gravitational field (but without a magnetic field) it is assumed that a wave phenomenon may be described in terms of a largely inert background medium and a disturbance. Thus, the density and pressure perturbations are first-order quantities, and the energy flux of the wave is a second-order quantity.

With the result by Anderson \& Athay for the flux divergence per gram in the region of the chromospheric temperature plateau we can check whether the basic assumptions on which the cutoff period rests are valid for the waves that heat the solar chromosphere. We find that each pulse of a $200 \mathrm{~s}$ wave dissipates an energy of about $1 \mathrm{eV}$ per particle. This exceeds the thermal energy per gram of the chromospheric gas, which is approximately $1 / 2 \mathrm{eV}$ per particle. Thus, for these waves, the wave energy is a zeroth-order quantity in the hydrodynamic equations, and hence the linearization is not allowed and the cutoff condition does not apply. There is therefore no a priori reason why the chromosphere should not be heated by waves with periods near the acoustic cutoff period.

\section{SUMMARY}

The chromosphere is identified with the atmosphere inside magnetic flux tubes. In the quiet sun below the $7000^{\circ} \mathrm{K}$ level, i.e., the layers traditionally referred to as the low and middle chromosphere, the gas is heated by compressional waves with periods mainly between $2 \mathrm{~min}$ and $4 \mathrm{~min}$. The heating mechanism in the high chromosphere, where hydrogen is completely ionized, may be different from that in the lower layers.

The energy dissipated by each wave pulse is comparable to the thermal energy of the chromospheric gas. Hence the cutoff condition that limits propagation of low-amplitude disturbances to waves with periods shorter than the cutoff period does not apply to these large-amplitude waves.

Estimates of the energy supplied to the low and middle chromosphere suggest that the $3 \mathrm{~min}$ waves can supply all the energy that is needed to heat the chromosphere to the 
observed temperatures. This conclusion is based on (1) the estimated energy flux in the compressional waves, which matches the observed chromospheric emission of the model VAL-C within a factor of about two, (2) the close agreement between the excess energy emitted by the $\mathrm{H}$ line in response to the dissipation by the observed compressional waves and the $\mathrm{H}$ line cooling rate in the empirical model VAL- $\mathrm{C},(3)$ on the estimate of the mechanical dissipation rate per unit mass implied by the empirical temperature curve, which is consistent with heating by acoustic waves, and (4) on the fact that weak-shock theory can approximately account for the properties of the observed compressional waves. The weight of this evidence implies that the chromosphere is heated only by these longperiod waves and that no other heating mechanism makes a significant contribution to the energy input of the chromosphere.

I thank Thomas R. Ayres, Franz-Ludwig Deubner, Jan Stenflo and Harold Zirin for comments on the chromospheric model. This work was supported by NASA grant NAGW1568 .

\section{REFERENCES}

Anderson, L. S. \& Athay, R. G. 1989, Astrophys. J., 336, 1089.

Athay, R. G. 1976, The Solar Chromosphere and Corona, Reidel Pub. Co., Dordrecht, Holland.

Ayres, T. R. 1981, Astrophys. J., 244, 1064.

Ayres, T. R. \& Testerman, L. 1981, Astrophys. J., 245, 1124.

Ayres, T. R., Testerman, L. \& Brault, J. W. 1986, Astrophys. J., 304, 542.

Bhatnagar, A. \& Tanaka, K. 1972, Solar Phys. 24, 87.

Biermann, L. 1946, Naturwiss., 25, 161.

Bray, R. J. \& Loughhead, R. E. 1974, The Solar Chromosphere, Chapman and Hall, London.

Cram, L. 1974, in: I.A.U. Symp. No. 56, Chromospheric Fine Structure, R. G. Athay ed., Reidel, Dordrecht Holland, 51.

1987 in: Cool Stars, Stellar Systems, and the Sun, J. L. Linsky and R. E. Stencel eds., Springer Verlag, Berlin, 123.

Cram, L. E. \& Damé, L. 1983, Astroph. J. 272, 355.

Damé, L. 1984, in: Small-Scale Dynamical Processes in Quiet Stellar Atmospheres, S. L. Keil ed., Sacramento Peak, p54.

Defouw, R. J. 1976, Astroph. J. 209, 266.

Foing, B. \& Bonnet, R. M. 1984, Astrophys. J., 279, 848.

Kalkofen, W. 1989, Astroph. J., subm.

Lindsey, C. E Roellig, T. 1987, Astroph. J. 313, 877.

Liu, S.-Y. 1973, Solar Phys. 31, 127.

1974, Ap. J. 189, 359.

Narain, U. \& Ulmschneider, P. 1989, Space Sci. Rev., subm.

Orrall, F. Q., 1966, Astroph. J. 143, 917.

Schatzman, E. \& Souffrin, P. 1967, Ann. Rev. Astron. Astroph., 5, 67.

Schwarzschild, M. 1948, Astrophys. J., 107, 1.

Simon, M. \& Shimabukuro, F. I. 1971, Astroph. J. 168, 525.

Ulmschneider, P. 1970, Solar Phys. 12, 403.

Vernazza, J. E., Avrett, E. H. \& Loeser, R. 1981, Astroph. J. Suppl., 45, 635, (VAL).

Yudin, O. I. 1968, Sov. Phys. - Doklady, 13, 503.

Zirin, H. 1988, Astrophysics of the Sun, Cambridge University Press, Cambridge, UK. 